\title{
¿CÓMO EXPLICAN LOS NIÑOS Y ADOLESCENTES EL CÁNCER AVANZADO O TERMINAL DE SUS PADRES?
}

\author{
HOW DO CHILDREN AND ADOLESCENTS EXPLAIN ABOUT ADVANCED OR \\ TERMINAL CANCER FROM THEIR PARENTS?
}

Anna Rodríguez Morera

Institut Català d'Oncologia. Facultat de Medicina. Universitat de Girona.

Resumen

Introducción: La comunicación entre padres e hijos es un proceso complejo, interactuando entre si diversos factores: desarrollo del niño, estilo educativo familiar y valores culturales. El objetivo del estudio fue aproximarse a las descripciones que los hijos hacen del cáncer avanzado o terminal de sus padres.

Métodos: Estudio exploratorio cualitativo con muestra de 74 niños y adolescentes de edades entre 5 y 18 años, junto a sus progenitores enfermos y sus progenitores sanos. Mediante entrevista semiestructurada se registró la información que recibían los hijos de la enfermedad y su comprensión.

Resultados: La cantidad y el tipo de información que explicaban ambos padres a sus hijos estaba condicionada por el dilema de contar o no el diagnóstico de cáncer. Los adolescentes recibían más información que los niños, sin diferencias entre sexos. La mayoría de niños y adolescentes daban explicaciones adaptativas, independientemente de su edad y sexo, siendo las definiciones de los adolescentes más técnicas y médicas.

Conclusiones: Los equipos de Oncología deberían integrar de manera preventiva, sistemática y escrupulosamente específica la atención psicológica para todos los padres con cáncer avanzado o terminal y con hijos menores.

Palabras clave: Comunicación, cáncer padres, cuidados paliativos, niños, adolescentes.

\section{Abstract}

Background: Communication between parents and children is a complex process, many factors interact: child's development, family education style and cultural values. Objective of this study was get closer to the descriptions made by children about their parents advanced or terminal cancer.

Methods: Exploratory qualitative study, with a sample of 74 children and adolescents, aged between 5 and 18 years, jointly with their parents having cancer and also with their healthy parents. Information received by children about the illness and his comprehension, where registered trough semi-structured interviews.

Results: The amount and type of information explained from both parents to their children was conditioned by the dilemma of explaining or not the diagnosis of cancer. Adolescents received more information than children, without observing differences between sexes. The majority of children and adolescents descrived adaptively the disease from their parents, regardless of their age and sex, being adolescents who used more technical and medical definitions.

Conclusions: Oncology teams should integrate the psychological care in a preventive, systematic and strictly specific way for all the parents with advanced or terminal cancer having children.

Key words: Communication, parental cancer, palliative care, children, adolescents.

\section{Correspondencia:}




\section{INTRODUCCIÓN}

La manera de comunicar la enfermedad a los hijos es un proceso complejo, heterogéneo y condicionado por diversos factores: desarrollo del niño, actitud familiar y aspectos culturales ${ }^{(1)}$.

En relación al desarrollo del niño, debemos puntualizar que la adquisición del concepto de enfermedad en los niños no depende únicamente de la información que se les facilita, si no que surge de la interacción entre la información y el nivel cognitivo del niño ${ }^{(2)}$. Aunque a cortas edades los niños ya perciben de manera vaga la noción de gravedad $^{(3)}$ no será hasta los 9 o 10 años que los niños comprenden el cáncer como un proceso de enfermedad grave y con posibilidades de morir, aumentando la precisión de sus causas y su evolución con la edad ${ }^{(2)}$. Asimismo, el concepto de muerte se adquiere conceptualmente de una forma similar ${ }^{(4)}$. Entendemos pues la percepción subjetiva de gravedad como un mediador del proceso de adaptación del niño o adolescente a la situación $^{(5)}$.

En este sentido debemos considerar que algunos niños y adolescentes manifiestan, más los adolescentes que los niños ${ }^{(3)}$, que no tienen las necesidades de información y de soporte satisfechas( ${ }^{(6,7)}$, y curiosamente pocas veces trasladan abiertamente este tipo de preocupaciones a sus padres ${ }^{(8,9)}$.

Relativo al aspecto familiar, a menudo la relación comunicativa dentro de la familia determina las estrategias adaptativas individuales de cada uno de sus miem$\operatorname{bros}^{(10-13)}$, manteniéndose el estilo de comunicación bastante estable durante toda la enfermedad ${ }^{(14)}$. No podemos obviar que hablar sobre la enfermedad de manera efectiva con los niños no es una tarea simple para los padres y no queda restringida únicamente a dar información médica, si no que implica aportar soporte y transmitir sentimientos de implicación ${ }^{(8,15)}$.
Y finalmente a nivel cultural, convenir que actualmente en el sur de Europa se acostumbra a proteger a los niños del sufrimiento emocional asociado a las malas noticias, presuponiendo que no están suficientemente capacitados para entender las informaciones dolorosas, arrebatando de manera poco consciente, su derecho a la autonomía ${ }^{(16)}$.

El principal objetivo del trabajo fue explorar como comprendían los niños y adolescentes el cáncer avanzado o terminal de sus padres, añadiendo de forma complementaria la descripción de cómo los padres informaban a sus hijos. De manera tácita, se reivindicó la importancia del tema de la comunicación del cáncer entre padres e hijos dentro de los equipos de oncología.

\section{MÉTODO}

\section{Participantes}

Se realizó un estudio cualitativo de quince meses de duración, entre noviembre de 2011 y enero de 2013, con una muestra de 74 niños y adolescentes, de edades comprendidas entre 5 y 18 años, junto a sus progenitores enfermos de cáncer avanzado o terminal y sus progenitores sanos. Los pacientes y sus hijos fueron reclutados indistintamente de los servicios de Hospitalización y de Consultas externas del Institut Català d'Oncologia de Girona. La participación fue voluntaria, tanto para padres enfermos como para sus parejas y sus hijos, firmando el correspondiente Consentimiento Informado todos los participantes con edad igual o superior a 12 años. Se incluyeron aquellos progenitores sanos que compartían con el paciente la paternidad/maternidad de los hijos menores, con la exclusión de aquellos progenitores con los que el paciente no refería tener ningún tipo de relación. El estudio estuvo evaluado y aprobado 
por el Comitè Ètic i d'Investigació Clínica de l'Hospital Universitari de Girona Doctor Josep Trueta.

\section{Instrumentos}

Se elaboró de forma exclusiva para el estudio una breve entrevista semiestructurada para progenitores y otra para niños y adolescentes. En la figura 1 se detallan las preguntas principales de las entrevistas a progenitores y a menores.

\section{Figura 1. Entrevistas a pacientes y progenitores sanos, niños y adolescentes}

\section{ENTREVISTA PROGENITORES}

¿Qué información tiene tu hijo/a de la enfermedad?

¿Por qué?

ENTREVISTA NIÑOS Y ADOLESCENTES

¿Qué le pasa a tu papá/mamá?

Explícame alguna cosa de lo que crees que le pasa. Puedes explicarme lo que quieras.

\section{Procedimiento}

En primer lugar, se informaba al paciente de los objetivos y del proceso de recogida de datos del estudio, y si este aceptaba participar, se preguntaba con su autorización a los familiares. A través de una entrevista a ambos progenitores por separado se anotaron por escrito las respuestas directas en referencia a la información del cáncer que tenían sus hijos. Por otra parte, y en un segundo momento, se propuso a los menores expresar de forma libre y mediante una redacción escrita o narración oral sus conocimientos y percepciones acerca de la enfermedad de sus padres. Con el conjunto de todas las respuestas, se elaboró una única matriz de datos, que mediante análisis cualitativo permitió la extracción de las principales categorías de respuesta expresadas por progenitores y hijos. Los datos cuantitativos fueron analizados usando el paquete estadístico SPSS 17.0, obteniéndose así los datos descriptivos y las relaciones entre variables numéricas y categoriales.

\section{RESULTADOS}

La muestra estuvo formada por $74 \mathrm{ni}-$ ños y adolescentes, de edades comprendidas entre 5 y 18 años $(M=11,2 ; D E=4,2)$, de los cuales $36(31,6 \%)$ eran niños y 38 $(33,3 \%)$ niñas, y por grupos de edad, 39 $(53 \%)$ eran niños y $35(47 \%)$ adolescentes, $31(41,9 \%)$ tenían el padre enfermo y $43(58,1 \%)$ la madre enferma, junto a sus 48 progenitores con cáncer avanzado o terminal y 41 progenitores sanos. En la Tabla 1 se detallan los parámetros sociodemográficos y clínicos de la muestra de pacientes del estudio.

La mayoría de pacientes y progenitores sanos referían que sus hijos tenían la información adecuada, justificando la cantidad y el tipo de información transmitida a los menores con los siguientes argumentos: el dilema de dar o no el diagnóstico de cáncer, la voluntad de querer proteger del sufrimiento de las malas noticias, la presuposición de la capacidad de comprensión del tema, la edad del menor y el interés que mostraba este por saber de la enfermedad. Los mismos motivos podían originar actitudes totalmente opuestas y maneras de comunicar diferenciadas, por ejemplo, con la misma justificación de la edad se podía informar o no a un niño, y con la misma intención de protección se podía informar o no informar al hijo. Más de la mitad de los niños y adolescentes de la muestra nunca habían recibido de los padres la información de que la enfermedad era un cáncer, limitándose en muchos casos la información a la palabra enferme- 
Tabla 1. Datos sociodemográficos y clínicos de la muestra de pacientes

\begin{tabular}{|c|c|c|c|}
\hline $\begin{array}{l}\text { Parámetros } \\
\text { sociodemográficos }\end{array}$ & Valor & Frecuencia (\%) & \\
\hline \multirow[t]{2}{*}{ Género } & Hombre & $19(39,6 \%)$ & \\
\hline & Mujer & $29(60,4 \%)$ & \\
\hline \multirow[t]{3}{*}{ Estado civil } & Casado/a o con pareja & $41(85,4 \%)$ & \\
\hline & Separado/a/Divorciado/a & $6(1,5 \%)$ & \\
\hline & Viudo/a & $1(2,1 \%)$ & \\
\hline \multirow[t]{4}{*}{ Núcleo de convivencia } & Familia nuclear & $36(75 \%)$ & \\
\hline & Familia monoparental & $6(12,5 \%)$ & \\
\hline & Familia reconstituida & $6(12,5 \%)$ & \\
\hline & Media (DE) & Mediana (Mín - Máx) & $\begin{array}{c}\text { Percentil } \\
25-75\end{array}$ \\
\hline Edad & $45,4(6,8)$ & $44(32-63)$ & $41-49$ \\
\hline Parámetros clínicos & Valor & Frecuencia (\%) & \\
\hline Diagnóstico oncológico & Estómago & $3(6,3 \%)$ & \\
\hline \multirow[t]{9}{*}{ principal } & Colon-Recto-Sigma & $6(12,5 \%)$ & \\
\hline & Pulmón & $9(18,8 \%)$ & \\
\hline & Mesotelioma & $1(2,1 \%)$ & \\
\hline & Mama & $13(27,1 \%)$ & \\
\hline & Útero-Ovario & $5(10,5 \%)$ & \\
\hline & Próstata & $1(2,1 \%)$ & \\
\hline & Riñón & $1(2,1 \%)$ & \\
\hline & Cerebro & $7(14,6 \%)$ & \\
\hline & Onco-hematológico & $2(4,2 \%)$ & \\
\hline \multirow[t]{4}{*}{ Grado de enfermedad } & $\begin{array}{l}\text { Enfermedad localmente } \\
\text { avanzada }\end{array}$ & $12(25 \%)$ & \\
\hline & Enfermedad diseminada & $14(29,2 \%)$ & \\
\hline & Enfermedad terminal & $21(43,8 \%)$ & \\
\hline & Situación de últimos días & $1(2,1 \%)$ & \\
\hline \multirow[t]{3}{*}{ Situación final estudio } & Enfermedad en proceso & $21(43,8 \%)$ & \\
\hline & Fallecimiento & $27(56,3 \%)$ & \\
\hline & Media (DE) & Mediana & $\begin{array}{l}\text { Percentil } \\
25-75\end{array}$ \\
\hline $\begin{array}{l}\text { Tiempo de enfermedad } \\
\text { (meses) }\end{array}$ & $17,7(23,9)$ & 12 & $2,5-20,8$ \\
\hline
\end{tabular}

dad. Se observó también una diferencia significativa en ambos progenitores a la hora de informar del diagnóstico de cáncer a sus hijos, siendo más frecuente dar esta información a los hijos adolescentes. En las tablas 2 y 3 se detallan los porcentajes de las justificaciones de pacientes y progenitores sanos al informar de la enfermedad a los hijos, según la edad del menor (niños y adolescentes), y según el sexo del 
menor (niños y niñas), y las relaciones entre dichas variables usando la prueba Chi cuadrado, o en caso de no aplicabilidad el Test exacto de Fisher (casillas con valor absoluto $\mathrm{p} \leq 0,05)$. hija de 14 años decía: "Sólo sabe que está enfermo, pero entiende que no puede hacer muchas cosas". A la vez, el $60 \%$ mantenían en sus justificaciones una actitud de protección del hijo, como la de un pacien-

\section{Tabla 2. Porcentajes de frecuencia de las categorías de respuesta de pacientes y} progenitores sanos según la edad del menor, y relación entre variables

\begin{tabular}{|c|c|c|c|c|c|c|c|c|c|c|}
\hline \multirow{2}{*}{$\begin{array}{l}\text { SI se informa } \\
\text { por motivo de: } \\
\text { (porcentajes) }\end{array}$} & \multicolumn{2}{|c|}{$\begin{array}{l}\text { Edad } \\
\text { del niño }\end{array}$} & \multicolumn{2}{|c|}{$\begin{array}{l}\text { Interés } \\
\text { del niño }\end{array}$} & \multicolumn{2}{|c|}{$\begin{array}{l}\text { Capacidad } \\
\text { del niño }\end{array}$} & \multicolumn{2}{|c|}{$\begin{array}{l}\text { Protección } \\
\text { del niño }\end{array}$} & \multicolumn{2}{|c|}{$\begin{array}{l}\text { Diagnóstico de } \\
\text { cáncer }\end{array}$} \\
\hline & $\underset{Z}{Z}$ & 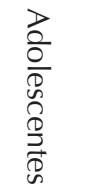 & $\underset{Z}{Z}$ & 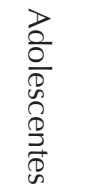 & $\underset{Z}{Z}$ & 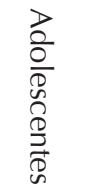 & $\underset{Z}{Z}$ & 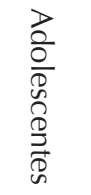 & $\underset{z}{Z}$ & 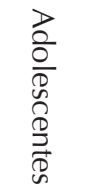 \\
\hline Pacientes & $5,1^{*}$ & $22,9^{*}$ & 17,9 & 37,1 & $17,9^{*}$ & $31,4^{*}$ & 23,1 & 42,9 & $23,1^{* *}$ & $54,3^{* *}$ \\
\hline Progenitores sanos & $15,6^{*}$ & $22,2^{*}$ & 21,9 & 40,7 & 25 & 25,9 & 21,9 & 33,3 & $18,8^{* *}$ & $63^{* *}$ \\
\hline
\end{tabular}

$p<0,05 ; " p<0,01$

Tabla 3. Porcentajes de frecuencia de las categorías de pacientes y progenitores sanos según el sexo del menor, y relación entre variables

\begin{tabular}{|c|c|c|c|c|c|c|c|c|c|c|}
\hline \multirow{2}{*}{$\begin{array}{l}\text { SI se informa } \\
\text { por motivo de: } \\
\text { (porcentajes) }\end{array}$} & \multicolumn{2}{|c|}{$\begin{array}{c}\text { Edad } \\
\text { del niño/a }\end{array}$} & \multicolumn{2}{|c|}{$\begin{array}{c}\text { Interés } \\
\text { del niño/a }\end{array}$} & \multicolumn{2}{|c|}{$\begin{array}{l}\text { Capacidad } \\
\text { del niño/a }\end{array}$} & \multicolumn{2}{|c|}{$\begin{array}{l}\text { Protección } \\
\text { del niño/a }\end{array}$} & \multicolumn{2}{|c|}{$\begin{array}{l}\text { Diagnóstico de } \\
\text { cáncer }\end{array}$} \\
\hline & $\begin{array}{l}\text { Z } \\
\text { soi } \\
\text { O্ }\end{array}$ & $\begin{array}{l}Z \\
\text { Zi } \\
\text { in } \\
\text { in }\end{array}$ & $\begin{array}{l}Z \\
\text { Z̃ } \\
\text { O } \\
\text { in }\end{array}$ & $\begin{array}{l}Z \\
\text { Zi } \\
\text { 品 }\end{array}$ & $\begin{array}{l}Z \\
\text { Z̄i } \\
\text { O }\end{array}$ & $\begin{array}{l}Z \\
\text { Zi } \\
\text { in } \\
\text { in }\end{array}$ & $\begin{array}{l}Z \\
\text { Z̄i } \\
\text { O্ }\end{array}$ & $\begin{array}{l}Z \\
\text { Żi } \\
\text { in }\end{array}$ & $\begin{array}{l}Z \\
\text { Z̃ } \\
\text { O } \\
\text { in }\end{array}$ & 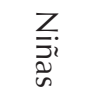 \\
\hline Pacientes & 13,9 & 13,2 & 27,8 & 26,3 & 25 & 23,7 & 36,1 & 28,9 & 36,1 & 39,5 \\
\hline Progenitores sanos & 16,7 & 20,7 & 33,3 & 27,6 & 20 & 31 & 33,3 & 20,7 & 40 & 37,9 \\
\hline
\end{tabular}

" $p<0,05 ; " p<0,01$

Los progenitores, en igual proporción pacientes y progenitores sanos, tenían la preocupación común de informar o no informar del diagnóstico de cáncer (por ejemplo un paciente con un hijo de 5 años manifestaba: "No le he dicho la palabra cáncer, le he dicho lo del bultito y le he dicho que voy al hospital a ponerme la medicina." y un progenitor sano con una te con una niña de 9 años que expresaba: "Prefiero que no se preocupe en exceso, tiene poca información para protegerla.", y un progenitor sano con una hija de 12 años defendía: "No quiero que sufra, hace cosa que sufra una niña tan pequeña". La mitad de pacientes y progenitores sanos informaban según presuponían al hijo capaz para comprender la enfermedad, por 
ejemplo, un paciente con una niña de 7 años apoyaba su decisión diciendo: "No tiene mentalmente madurez para entender la enfermedad, me ve como siempre", y un progenitor sano con una niña de 10 años decía simplemente: "Se conforma con jugar, él no comprende lo que pasa". Con menor frecuencia, aunque no despreciable, los progenitores valoraban el interés del menor antes de informar, por ejemplo con argumentos como el de un paciente con un niño de 11 años que concluía: "Si no pregunta, no tienes que hacer nada. No quiere saber", y un progenitor sano con una hija de 9 años matizaba: "Hemos contestado a sus preguntas, según su curiosidad". La edad del menor también era considerada de manera explícita en algunos progenitores, por ejemplo un paciente con un hijo de 15 años sostenía: "No tiene edad para saber estas cosas", y un progenitor sano con un niño de 8 años decía: "Por la edad que tiene no puede conocerlo todo".

Un total de 51 hijos, $24(47,1 \%)$ niños y 27 (52,9\%) adolescentes, y divididos por sexos, 29 (56,9\%) niños y 22 $(43,1 \%)$ niñas, explicaron la enfermedad de los padres. Los niños y adolescentes definían la enfermedad usando habitualmente términos médicos, por ejemplo un niño de 9 años decía: "A la mama se le tapó un tubo del hígado y tuvieron que desatascarlo con un tubo delgado como un cabello" y por ejemplo una adolescente de 17 años expresaba: "Mi madre tiene cáncer, exactamente en la pelvis, con metástasis en uréteres, riñones y cueIlo de matriz. Está terminal, estadio 4B, el máximo". También era habitual el uso de percepciones subjetivas tanto en niños como en adolescentes como por ejemplo un niño de 5 años contaba: "Antes le dolía la cabeza pero ahora se está curando un poco, pero está igual de mal" y una adolescente de 18 años: "Los tratamientos son muy fuertes y esto provoca que la persona cambie. Mi madre ha cambiado des del diagnóstico". Los pensamientos desiderativos o expresión de deseos era menos frecuente y se manifestaba con frases como: "Espero que se cure" de un niño de 11 años, o como la de un adolescente de 13 años que decía: "Sé que no tiene cura pero quiero que tenga fuerzas para seguir viviendo muchos años". Las manifestaciones que hacían referencia a la actitud de cuidar eran poco frecuentes, y se proponían como: "Está enferma y tenemos que cuidarla mucho" de un niña de 6 años o como: "Mientras mi madre no está, trato que mis hermanos no estén tristes" de una adolescente de 15 años. Algunos niños usaban la palabra cáncer o muerte en su redacción, por ejemplo una niña de 11 años expresaba: "Depende del día está mejor o peor, pero no se cura. Al final mi padre me contó que mamá tenía cáncer" y un adolescente de 18 años decía: "Tiene cáncer en los huesos por culpa de una metástasis que le pasó cuando hace años tubo cáncer de mama". Cuatro de los participantes $(7,8 \%)$ respondieron con clara evitación y sin querer hablar del tema, dando respuestas evasivas cómo las de una niña de 10 años: "No le pasa nada" y 5 participantes $(9,8 \%)$ argumentaron falsas creencias o errores, por ejemplo una adolescente de 15 años decía: "Ahora dicen que tiene una infección en el hueso". Por su falta de ajuste con la realidad, estos tipos de respuesta se consideraron explicaciones no adaptativas. En la tabla 4 podemos ver los porcentajes de frecuencia de las argumentaciones adaptativas de los niños y adolescentes al explicar la enfermedad de los padres, analizados por grupos de edad y sexo con la prueba Chi cuadrado (g.l.=1) o el Test exacto de Fisher (en caso de casillas con valor absoluto $\mathrm{p} \leq 0,05$ ).

Finalmente, agrupando el conjunto de respuestas en una sola variable dicotómica: Explicación adaptada (sin evitación ni 
errores de argumentación) y Explicación no adaptada (con evitación o errores en argumentación). La tabla 5 muestra algunos ejemplos de cada tipo de explicación y en la tabla 6 se describe la relación entre la variable dicotómica Explicación adaptada / Explicación no adaptada con las variables sociodemográficas edad y sexo mediante el Test exacto de Fisher (casillas con valor absoluto $\mathrm{p} \leq 0,05)$.

\section{Tabla 4. Frecuencias y porcentajes de las categorías adaptativas expresadas por niños y adolescentes al definir la enfermedad de los padres. Relación entre variables}

\begin{tabular}{|c|c|c|c|c|c|c|}
\hline Según edad & $\begin{array}{l}\text { Explicación } \\
\text { médica }^{*}\end{array}$ & $\begin{array}{l}\text { Percepciones } \\
\text { subjetivas }\end{array}$ & $\begin{array}{l}\text { Pensamientos } \\
\text { desiderativos }\end{array}$ & $\begin{array}{l}\text { Actitud } \\
\text { de cuidar }\end{array}$ & $\begin{array}{c}\text { Usar } \\
\text { cáncer }\end{array}$ & $\begin{array}{c}\text { Usar } \\
\text { muerte * }\end{array}$ \\
\hline Niños & $16(66,7 \%)$ & $16(66,7 \%)$ & $7(29,2 \%)$ & $4(16,7 \%)$ & $3(12,5 \%)$ & $1(4,2 \%)$ \\
\hline Adolescentes & $25(92,6 \%)$ & $23(85,2 \%)$ & $14(51,9 \%)$ & $9(33,3 \%)$ & $\begin{array}{c}18 \\
(66,7 \%)\end{array}$ & $\begin{array}{c}9 \\
(33,3 \%)\end{array}$ \\
\hline Según sexo & $\begin{array}{l}\text { Explicación } \\
\text { médica }\end{array}$ & $\begin{array}{l}\text { Percepciones } \\
\text { subjetivas }\end{array}$ & $\begin{array}{c}\text { Pensamientos } \\
\text { desiderativos }\end{array}$ & $\begin{array}{l}\text { Actitud } \\
\text { de cuidar }\end{array}$ & $\begin{array}{l}\text { Usar } \\
\text { cáncer }\end{array}$ & $\begin{array}{l}\text { Usar } \\
\text { muerte }\end{array}$ \\
\hline Niños & $25(86,2 \%)$ & $20(69 \%)$ & $11(37,9 \%)$ & $6(20,7 \%)$ & $9(31 \%)$ & $\begin{array}{c}5 \\
(17,2 \%)\end{array}$ \\
\hline Niñas & $16(72,7 \%$ & $19(86,4 \%)$ & $10(45,5 \%)$ & $7(31,8 \%)$ & $\begin{array}{c}12 \\
(54,5 \%)\end{array}$ & $\begin{array}{c}5 \\
(22,7 \%)\end{array}$ \\
\hline
\end{tabular}

" $p<0,05 ;{ }^{* *} p<0,01$

\section{Tabla 5. Ejemplos de Explicaciones adaptativas y Explicaciones no adaptativas de los niños y adolescentes}

\section{EXPLICACIONES ADAPTATIVAS}

Le están operando la cabeza, antes le dolía la cabeza pero ahora se está curando un poco... pero está igual de mal. (niño de 5 años con padre enfermo)

Está enferma, me preocupa que le pase algo. (niño de 12 años con madre enferma)

Espero que dentro de poco venga, así celebramos la Navidad juntos, sé que no tiene cura pero quiero que tenga fuerzas para seguir viviendo muchos años.

(niño de 13 años con padre enfermo)

Que tiene cáncer, tiene un tumor en el pecho y ya se lo han sacado. Pues... que tiene un tumor. (niño de 15 años con madre enferma)

Que tiene un tumor en la espalda y eso hace que no se encuentre bien, que esté débil, que no pueda hacer nada o tenga mal humor. No sé si es una enfermedad muy grave que pueda morir o si solo es dura pero se cura. (niño de 16 años con padre enfermo)

\section{EXPLICACIONES NO ADAPTATIVAS}

La mamá se encuentra mal porqué antes tenía un bicho en la teta.

(niña de 5 años con madre enferma)

No le pasa nada. (niña de 10 años con madre enferma)

Ahora dicen que tiene una infección en el hueso. (niña de 15 años con madre enferma) 


\section{Tabla 6. Frecuencias y porcentajes de la variable dicotómica Explicación adapta- da o Explicación no adaptada, según edad y sexo. Relación entre variables.}

\begin{tabular}{|cccc|}
\hline Según edad & $\begin{array}{c}\text { Explicación } \\
\text { adaptada }\end{array}$ & $\begin{array}{c}\text { Explicación } \\
\text { no adaptada }\end{array}$ & $\begin{array}{c}\text { Test exacto } \\
\text { de Fisher }\end{array}$ \\
Adolescentes & $\begin{array}{c}23(79,2 \%) \\
5(20,8 \%)\end{array}$ & $p>0,05$ \\
Según sexo & $\begin{array}{c}\text { Explicación } \\
\text { adaptada }\end{array}$ & $\begin{array}{c}\text { Explicación } \\
\text { no adaptada }\end{array}$ & $\begin{array}{c}\text { Test exacto } \\
\text { de Fisher }\end{array}$ \\
Niños & $25(86,2 \%)$ & $4(13,8 \%)$ & $p>0,05$ \\
Niñas & $17(77,3 \%)$ & $5(22,7 \%)$ & \\
\hline
\end{tabular}

Observamos que más del $80 \%$ de niños y adolescentes, aunque de diversa forma $y$ cada uno a su manera, realizaban una expresión congruente y adaptada de la situación de enfermedad de sus padres. No se encontró relación significativa entre el tipo de explicación del menor y las variables sexo y edad, es decir, niños y adolescentes podían expresar de manera adaptada o no adaptada la enfermedad de sus padres; y entre niños y niñas de cualquier edad, todos tenían las mismas proporciones de respuestas adaptadas y desadaptadas.

\section{DISCUSIÓN}

Pacientes y progenitores sanos informaban del diagnóstico de cáncer más habitualmente a sus hijos adolescentes que a sus hijos de edad escolar, no observándose diferencias significativas según el sexo del hijo, es decir, niños y niñas eran informados de manera similar del diagnóstico de cáncer. Estos resultados serían congruentes con estudios que igualaban la comunicación entre hijos e hijas ${ }^{(17)}$, y se apartarían de los estudios que consideraban diferencias de sexo en la comunicación entre padres e hijos ${ }^{(18)}$. Otras investigaciones han resaltado en el mismo sentido que los adolescentes reciben más información que los niños $^{(19)}$. De forma comprensible, la mejor capacidad cognitiva que presentan los adolescentes en comparación con los niños, facilitaría su comprensión de la información compleja, y permitiría que muchos padres hablasen abiertamente de la enfermedad con ellos. Cabe destacar que los padres cambiaban el concepto de protección a lo largo del crecimiento de su hijo, y se mostraban mucho más cautos a la hora de expresar emociones negativas ante los hijos más pequeños, actitud recomendada por autores como Christ y Christ ${ }^{(20)}$. Seguramente, los adolescentes informados de manera honesta y gradual, contribuyen a crear un entorno de confianza para muchos pacientes y progenitores sanos, y este hecho podría en un futuro generar mayor autoconfianza en el adolescente. $Y$ aunque las necesidades de información de los niños sean menores o menos evidentes para los padres ${ }^{(3,21)}$, existen y las padecen ${ }^{(22)}$ y tendríamos que aprender a detectarlas y considerarlas para su adecuada atención.

Los pacientes, consideraban más que los progenitores sanos la capacidad del adolescente a la hora de informar. Estos resultados indicarían que los pacientes, independientemente del sexo del hijo, buscarían una complicidad especial con los adolescentes, preferían compartir la 
información abiertamente con ellos. Por otra parte, los progenitores sanos mostraban puntos de vista más rígidos y informaban de manera más homogénea a todos sus hijos, niños y adolescentes. En este sentido, podríamos pensar como MacPherson ${ }^{(23)}$ que una parte importante de padres y madres sanas, abrumados por la situación de cuidar a los hijos y al paciente, y con el conflicto interno de atender sus necesidades y las de su familia, optarían por dar la información más simple y menos individualizada de la enfermedad a sus hijos. Los progenitores cuidadores, podrían intentar preservar el clima de normalidad y de bienestar dejando los temas difíciles sin hablar, con las consecuencias de una comunicación pobre con sus hijos sobre la enfermedad. Sería en este sentido recomendable que los equipos de oncología detectaran este tipo de situaciones y apoyaran profesionalmente a estos padres.

Los argumentos que los progenitores daban del porqué informar a los hijos eran los de comunicar o no el diagnóstico de cáncer, la intención de proteger del sufrimiento, el interés del hijo, la capacidad para comprender la enfermedad, y en menor frecuencia, la edad del menor. La gran mayoría de justificaciones de pacientes y progenitores sanos reflejaban el impacto emocional que la enfermedad había representado en sus vidas, y la presuposición de que sus hijos reaccionarían de igual manera al estrés que los adultos, con las mismas necesidades y los mismos miedos. Con todo, no se solía preguntar a los niños y adolescentes, y muchos padres acordaban unilateralmente cómo informar a los hijos del cáncer, pensando en la información más adecuada para cada hijo según sus convicciones, resultados congruentes con estudios previos ${ }^{(24)}$. Por este motivo, la información recibida por el menor era parcial y no siempre contestaba a sus preguntas y preocupaciones. En este sentido, recomendaríamos mantener una atención psicológica especializada con las familias de pacientes con hijos menores, con el fin de mejorar los resultados de la comunicación de la enfermedad y controlar el estrés que puede significar para todos no poder hablar del cáncer en casa ${ }^{(25)}$.

Los niños y adolescentes, en su mayoría, explicaban la enfermedad con términos médicos y percepciones subjetivas. Los adolescentes usaban significativamente más tecnicismos, más la palabra cáncer y la posibilidad de muerte. Los deseos y las actitudes de ayuda aparecían en las narraciones de niños y adolescentes de manera ocasional, siendo habitual expresar el deseo de curación. No se encontraron diferencias significativas entre sexos al definir la enfermedad de los padres, y las redacciones y narraciones de nuestra muestra de hijos fueron similares a las de niños y adolescentes de estudios con población anglosajona que habían recibido mayor información de la enfermedad. ${ }^{(12)}$ En definitiva, los niños y adolescentes utilizaban el vocabulario médico que habían escuchado de sus padres, ya que habitualmente los padres explicaban de esta forma la enfermedad, evitando los mensajes con excesiva emotividad. Posiblemente los hijos necesitarían como complemento una aproximación de la situación con términos más psicosociales y emocionales ${ }^{(15,25)}$, o simplemente, que validásemos su manera de ver y sentir la situación ${ }^{(20)}$, sin descuidar que no siempre toda expresión emocional resulta positiva para el bienestar del menor ${ }^{(27)}$. Recomendaríamos pues a los padres encontrar aquella dosis de información adecuada para cada hijo y para cada situación en concreto ${ }^{(28)}$.

En general, las explicaciones de los niños y adolescentes se consideraron adaptadas, puesto que no evitaban el tema ni daban explicaciones erróneas, sin diferencias entre edades y sexos. Estos resultados confirmarían las conclusiones de otros estudios pioneros ${ }^{(29)}$ y estudios más 
recientes $^{(3)}$ en que se evidenciaba que los niños ya a partir de los 5 años podían ser conscientes de alguna manera de que en casa estaban pasando cosas importantes. Asimismo, tendríamos que resaltar que aunque los niños y adolescentes puedan tener pistas del malestar familiar y sientan inquietudes por conocer la enfermedad de los padres, pocas veces acabarán preguntando abiertamente a los padres, adoptando, como los adultos, una actitud de protección hacia los padres inhibiendo la comunicación de miedos y preocupaciones dentro de casa, confundiendo aún más la interpretación que hacen sus padres acerca de su bienestar. ${ }^{(30)}$

Una de las principales limitaciones de este estudio fue analizar de manera global las categorías de respuesta de pacientes y progenitores, agrupando en el análisis argumentaciones con puntos de vista muy diferentes, sin diferenciar los tipos de actitud abierta o cerrada en referencia a informar a los hijos. Sería recomendable pues en futuras investigaciones focalizar la atención en como las familias orientan el tema de la comunicación, si de manera abierta o de manera cerrada, y de cómo evoluciona la comunicación durante el proceso de la enfermedad, y las consecuencias que podrían aparecer a largo plazo. De otra parte, tiene el valor añadido el hecho de estudiar un tema delicado en nuestra cultura y realizar una aproximación cualitativa que permite empezar a comprender los matices de la comunicación del cáncer en nuestras familias, tal y como recomiendan algunas revisiones actuales. ${ }^{(31)}$ Además, el análisis por edades y por sexos, permitirá la realización de futuras réplicas de la investigación y la comparación de resultados.

\section{CONCLUSIONES}

A modo de conclusión este estudio invita a que los equipos de oncología reflexionen en ayudar a muchos padres a comunicarse de manera más cómoda y efectiva con sus hijos durante las etapas finales de la enfermedad. De todas formas, se necesitan nuevos estudios empíricos y cualitativos en nuestra población para profundizar en el conocimiento de este tema tan complejo.

Agradecimientos: Dar las gracias a la Dirección de centro del Institut Català d'Oncologia por su colaboración en la realización del trabajo, a los miembros del CEIC del Hospital Universitari de Girona Doctor Josep Trueta por su revisión y aprobación del protocolo, y a todos los profesionales que han contribuido en la aportación desinteresada de ideas, soporte técnico y/o en el reclutamiento de pacientes. Y de manera muy especial, agradecer la colaboración de todos los participantes en el estudio. A todos, muchas gracias.

\section{REFERENCIAS BIBLIOGRÁFICAS}

1. Beale E, Sivesind D, Bruera E. Parents dying of cancer and their children. PaIliat Support Care 2004;2:387-93. Doi: 10.1017/S1478951504040519

2. Del Barrio C. La comprensión infantil de la enfermedad. Un estudio evolutivo. Barcelona: Anthropos, 1990.

3. Forrest G, Plumb C, Ziebland S, Stein A. Breast cancer in the family children's perceptions of their mother's cancer and its initial treatment: Qualitative study. BMJ 2006;332:998-1001. Doi: 10.1136\%2Fbmj.38793.567801.AE

4. Kissane DW, Zaider T. Bereavement. En: Hanks G, Cherny NI, Christakis N, Fallon M, Kaasa S, Portenoy RK, editors. Oxford Textbook of Palliative Medicine. 4th ed. New York: Oxford University Press; 2010. p.1483-501.

5. Compas BE, Worsham NL, Ey S, Howell DC. When mom or dad has cancer: II. Coping, cognitive appraisals, and psychologi- 
cal distress in children of cancer patients. Health Psychol 1996;15:167-75.

6. Finch A, Gibson F. How do young people find out about their parent's cancer diagnosis: A phenomenological study. Eur J Oncol Nurs 2009;13:213-22. Doi: 10.1016/j.ejon.2009.03.010

7. Kristjanson LJ, Chalmers KI, Woodgate R. Information and support needs of adolescent children of women with breast cancer. Oncol Nurs Forum 2004;31:111-9. Doi: 10.1188/04.ONF.111-119

8. Kennedy VL, Lloyd-Williams M. Information and communication when a parent has advanced cancer. J Affect Disord 2009;114:149-55. Doi: 10.1002/pon.1455

9. Schmitt F, Piha J, Helenius H, Baldus C, Kienbacher C, Steck B, et al. Multinational study of cancer pacients and their children: Factor associated with family functioning. J Clin Oncol 2008;26:587783. Doi: $10.1200 / \mathrm{JCO} .2007 .12 .8132$

10. Osborn T. The psychosocial impact of parental cancer on children and adolescents: a systematic review. Psychooncology 2007;16:101-26. Doi: 10.1002/pon.1113

11. Lindqvist B, Schimitt F, Santalahti $P$, Romer G, Piha J. Factors associated with the mental health of adolescents when a parent has cancer. Scand J Psychol 2007;48:345-51. Doi: 10.1111/j.14679450.2007.00573.x

12. Trastum M, Johansen M, Gubba L, Olesen $\mathrm{L}$, Romer G. Coping, social relations, and communication: A qualitative exploratory study of children of parents of cancer. Clin Child Psychol Psychiatry 2008;13:123-38. Doi:10.1177/1359104507086345

13. Vannatta K, Ramsey RR, Noll RB, Gerhardt CA. Associations of child adjustment with parent and family funtioning: comparison of families of women with and without breast cancer. J Dev Behav Pediatrics 2010;31:9-16. Doi: 10.1097/DBP. 0b013e3181c82a44

14. Gazemdam-Donofrio SM, Hoekstra HJ, van der Graaf WT, van de Wiel HB, Vis- ser A, Huizinga GA, Hoekstra-Weebers JE. Parent-child comunication patterns during the first year after a parent's cancer diagnosis: The effect on parents' functioning. Cancer 2009;115:4227-37. Doi: 10.1002/ cncr.24502

15. Bugge KE, Helseth $S$, Darbyshire $P$. Children's experiences of participation in a family support program when their parent has incurable cancer. Cancer Nurs 2008;31:426-34. Doi: 10.1097/01. NCC.0000339250.83571.b0

16. Comitè Bioètica Catalunya. La persona menor d'edat en l'àmbit de la salut. Barcelona: Comitè Bioètica Catalunya; 2009. [Acceso el 8 de enero de 2013]. Disponible en: http://www.pcb.ub.edu/bioeticaidret/archivos)

17. Barnes J, Kroll L, Burke O, Lee J, Burke O, Jones A, Stein A. Qualitative interview study of communication between parents and children about maternal breast cancer. BMJ 2000;321:479-81.

18. Huizinga GA, Visser A, van der Graaf WT, Hoekstra HJ, Hoekstra-Weebers JE. The quality of communication between parents and adolescent children in the case of parental cancer. Ann Oncol 2005;16:195661. Doi: 10.1093/annonc/mdi395

19. Rosenheim E, Reicher R. Informing children about a parent's terminal illness. J Child Psychol Psychiatry 1985;26,995-8.

20. Christ GH, Christ AE. Current approaches to helping children cope with a parent's terminal illness. CA A Cancer J Clin 2006;56:197-212. Doi: 10.3322/canjclin.56.4.197

21. Welch AS, Wadsworth ME, Compas BE. Adjustment of children and adolescents to parental cancer. Parents' and children's perspectives. Cancer 1996;77:1409-18.

22. Zahlis EH. The child's worries about the mother's breast cancer: sources of distress in school-age children. Oncol Nurs Forum 2001;28:1019-25.

23. MacPherson $\mathrm{C}$. Telling children their ill parent is dying: A study of the factors influen- 
cing the well parent. Mortality 2005; 10:11326. Doi: 10.1080/13576270500102872

24. Barnes J, Kroll L, Lee J, Burke O, Jones A, Stein A. Factors predicting communication about the diagnosis of maternal breast cancer to children. J Psychosom Res 2002;52:209-14. Doi: 10.1016/S00223999(02)00296-9

25. Huizinga GA, Visser A, van der Graaf WT, Hoekstra HJ, Stewart RE, Hoekstra-Weebers JE. Family-oriented multilevel study on the psychological functioning of adolescent children having a mother with cancer. Psychooncology 2011;20:730-7. Doi: 10.1002/pon.1779

26. Shands ME, Lewis FM, Zahlis EH. Mother and child interactions about the mother's breast cancer: An interview study. Oncol Nurs Forum 2000;27:77-85.

27. Christ G. Impact of development on children's mourning. Cancer Pract, 2000;8:72-81. Doi: 10.1046/j.15235394.2000.82005.x
28. Rodríguez-Morera A. Els nens i adolescents davant el càncer avançat o terminal dels pares. (Tesis doctoral). Bellaterra: Universidad Autònoma de Barcelona; 2013.

29. Siegel K, Mesagno FP, Karus D, Christ G, Banks K, Moyhihan R. Psychosocial adjustment of children wirh a terminally ill parent. J Am Acad Child Adolesc Psychiatry 1992;31:327-33. Doi: 10.1097/00004583-199203000-00022

30. Forrest G, Plumb C, Ziebland S, Stein A. Breast cancer in Young families: A qualitative interview study of fathers and their role and communication with their children foIlowing the diagnosis of maternal breast cancer. Psychooncology 2009;18:96-103. Doi: 10.1002/pon.1387

31. Visser A, Huizinga GA, van der Graaf WT, Hoekstra HJ, Hoekstra-Weebers JE. The impact of parental cancer on children and the family: A review of the literature. Cancer Treat Rev 2004;30:683-94. Doi: 10.1016/j.ctrv.2004.06.001 